\title{
DIRECT VENEER RESTORATION USING A NANO CERAMIC COMPOSITE MATERIAL: A CLINICAL REPORT
}

N.A. Yahya, K.H.D. Lim. Direct Veneer Restoration

Using A Nano Ceramic Composite Material: A Clinical Report. Annal Dent Univ Malaya 2008; 15(2): 89-93.

\begin{abstract}
Direct composite veneers can be used to mask tooth discolorations and/or to correct unaesthetic tooth forms and/or positions. However, these type of restorations are often regarded as one of the most challenging in aesthetic dentistry presumably due to the extent of natural tooth structure that must be recreated. This paper discusses easy application techniques and tips for Ceram.X Duo ${ }^{\mathrm{TM}}$, a nano ceramic composite restorative material. Its natural shading system allows the restoration of tooth with both dentine and enamel shade and transforms it into a final direct veneer restoration that mimics a natural tooth.
\end{abstract}

Key words: nano ceramic composite; Ceram.X Duo $^{\mathrm{TM}}$, direct veneer restoration, aesthetic

\section{INTRODUCTION}

The growing importance placed on aesthetics may result in an increased demand for cosmetic dental treatment (1). Over the years, we have seen that the patients' demand for treatment of unaesthetic anterior teeth is steadily rising. Accordingly, several treatment options have been proposed to restore the aesthetic appearance of the dentition. For many years, full crowns have been the most predictable and durable aesthetic restoration of anterior teeth (2). However, this approach is undoubtedly most invasive with substantial removal of large amounts of tooth substance and possible adverse effects on adjacent pulp and periodontal tissues.

The advancement in bonding capability to both enamel and dentine made with the introduction of multi-step total-etch adhesive systems (3-5), along with the development of high performance and more universally applicable resin composites has led to more conservation restorative adhesive techniques to deal with unaesthetic tooth appearance. Resin composite veneers can be used to mask tooth discolorations and/or to correct unaesthetic tooth forms and/or positions. Both direct and indirect bonding techniques can be used for this type of restoration. The direct veneer restoration is often regarded as one of the most challenging procedure in aesthetic dentistry presumably due to the extend
Case Report

N.A. Yahya', K.H.D. Lim ${ }^{2}$
${ }^{1}$ Senior Lecturer
Department of Conservative Dentistry
Faculty of Dentistry, University of Malaya
50603 Kuala Lumpur
Email: nazlin@um.edu.m]
${ }^{2}$ Dental Officer
Klinik Pakar Pergigian
Hospital Sultanah Aminah
Jalan Abu Bakar, 80100 Johor Bahru.

Corresponding author: Noor Azlin Yahya

of natural tooth structure that must be re-created. The clinical success of restorations depends on technical aspects, aesthetic result and performance in time.

In the past few years, Ceram.X Duo ${ }^{\text {TM }}$ (Dentsply De Trey, Konstanz, Germany), a nano ceramic restorative with double translucency for patients with higher aesthetic demands has been introduced into the market. It also has natural shading system by replacing lost dentine with dentine-like opacity and chroma and replacing enamel with enamel-like translucency. One of the advantages is this natural shading system enables the dentists to utilize various combinations of only 8 shades in order to meet specific patient needs. The purpose of this paper is to present a step-by-step protocol for predictable veneer restoration of discoloured anterior tooth/teeth with direct composite resin, Ceram.X Duo ${ }^{\mathrm{TM}}$, in a single appointment.

\section{CLINICAL REPORT}

A 24-year old female patient presented with a history of trauma on tooth 21 several years ago and the tooth was subsequently root treated. Internal bleaching was done in order to improve the colour however the patient was not satisfied with the outcome. Intraoral examination revealed that tooth 21 was restored with composite mesio-incisally (Fig. 1). The treatment objective was to improve the appearance of tooth 21 conservatively at minimal cost. The patient agreed to the treatment plan which was to place a direct veneer on tooth 21 with nano ceramic restorative material: Ceram.X Duo ${ }^{\mathrm{TM}}$ (Dentsply De Trey, Konstanz, Germany). The 


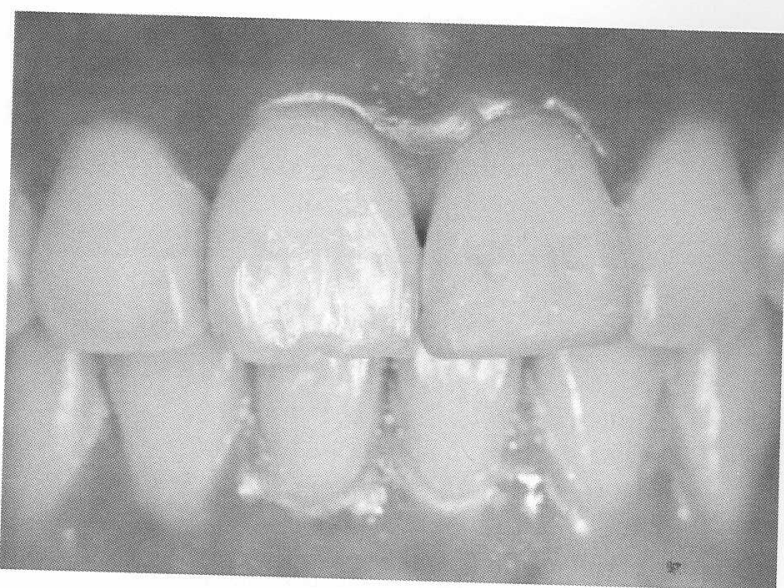

Figure 1: Preoperative view. A discoloured and poorly shaped tooth, 21 .

treatment procedure was as follows:

1. Shade was matched with VITA shade guide after the upper anterior teeth were cleaned with prophylaxis paste (Fig. 2). In this case, A1 VITA shade was the closest match (equivalent to E2 and D1 in Ceram.X Duo shade guide). Clinical tip: Shade should be selected in natural daylight with the teeth under moist condition. Eliminate any strong colour contrast such as lipstick.

2. Upon confirmation of the shade, retraction cord was placed into the gingival sulcus using a flat plastic instrument. Clinical tip: Place the cord in situation where rubber dam cannot be used. The cord will retract free gingiva away from the tooth and reduce the seepage of cervical fluid at the cervical area.

3. A depth cut bur (Komet no. 834.314.021, Gebr, Brasseler, Germany) was then used to create orientation grooves to facilitate labial reduction with a diamond chamfer bur (Komet no. 6856.314.018, Gebr, Brasseler, Germany) later (Fig 3 \& 4). Clinical tip: Align the burs to cut the grooves and labial reduction at two different planes following the crown contour.

4. After the 21 was prepared, it was then isolated from the adjacent teeth with a cellulose mylar strip (Fig. 5).

5. The labial surface of 21 was then etched with $36 \%$ phosphoric acid (De Trey ${ }^{\circledR}$ Conditioner 36, DENTSPLY DeTrey, Konstanz, Germany) prior to application of bonding agent, Prime \& Bond NT $^{\circledR}$ (DENTSPLY DeTrey, Konstanz, Germany) (Fig. $6 \& 7$ ).

6. The dentine shade composite (D1) was then placed and shaped (Fig. 8). A mammelon-like effect was produced incisally (Fig. 9). This layer was then light cured for 40 seconds with light curing unit, Spectrumò 800 (Dentsply Caulk, USA). Clinical tip: Sculpt the dentine shade

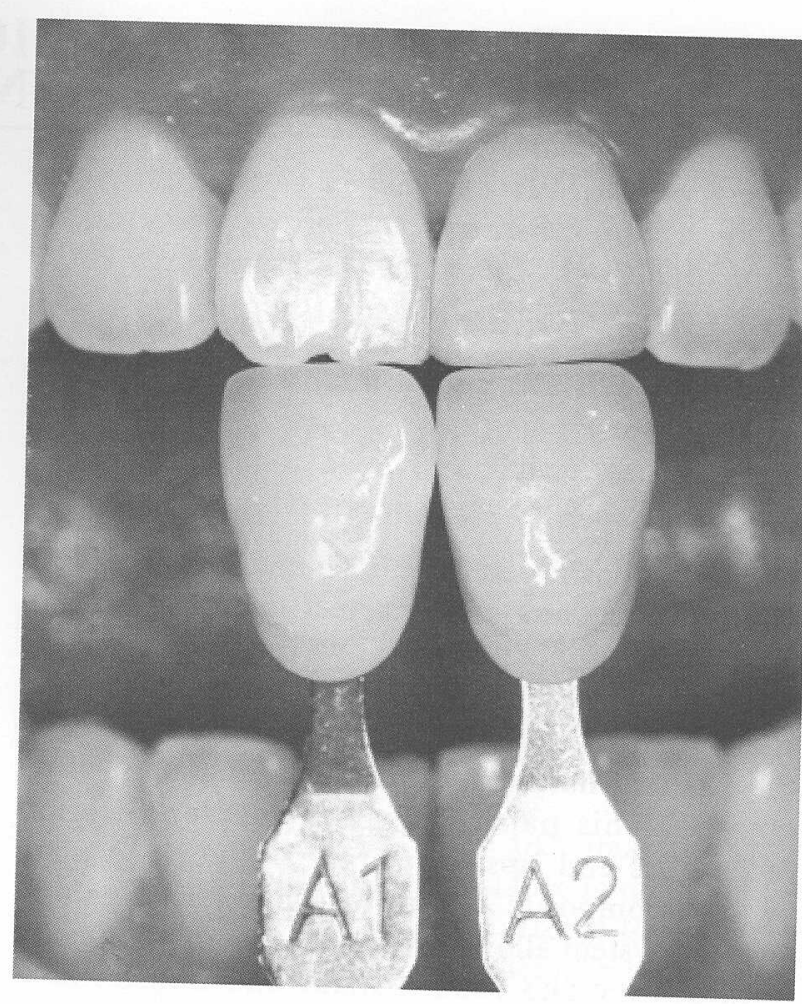

Figure 2: Shade selection.

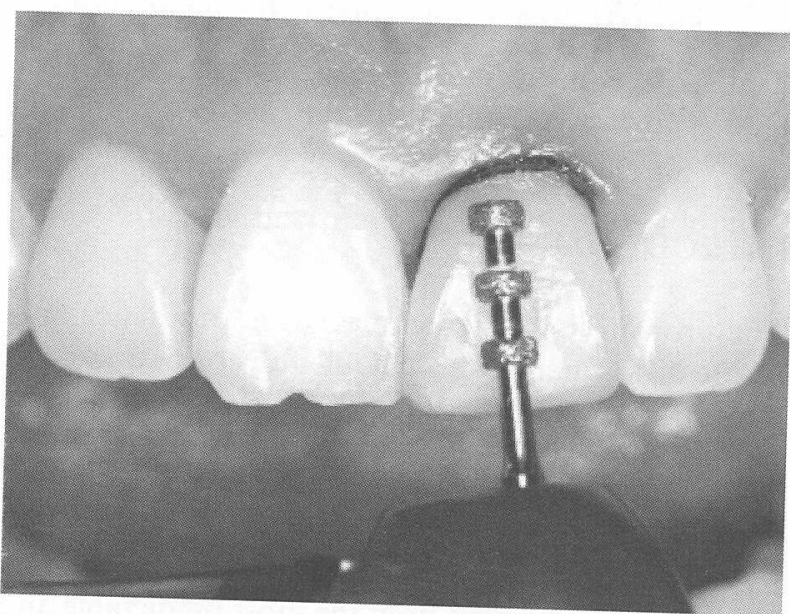

Figure 3: Orientation grooves were created with a depth cut bur.

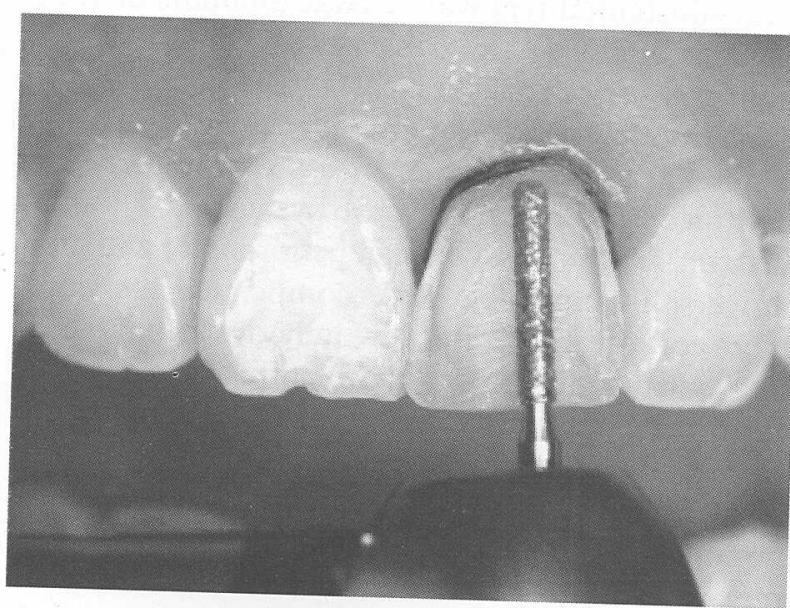

Figure 4: The labial surface of 21 was reduced using a chamfer diamond bur. 


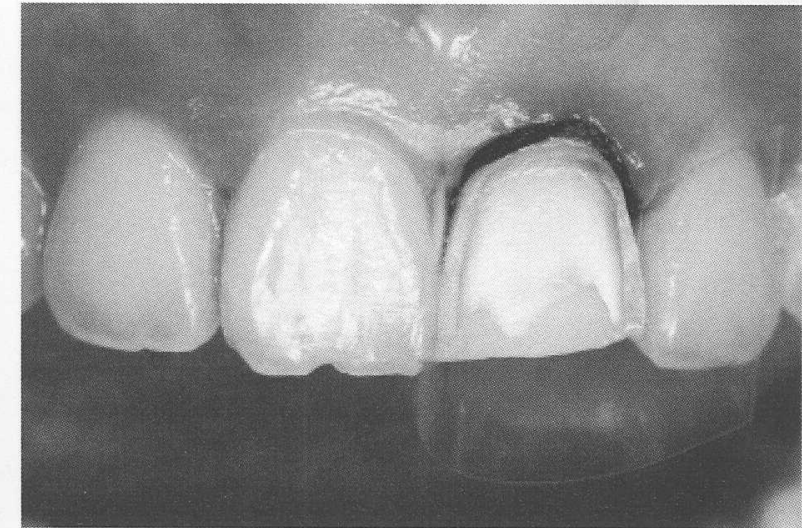

Figure 5: The prepared tooth was isolated from the adjacent teeth using a cellulose mylar strip.

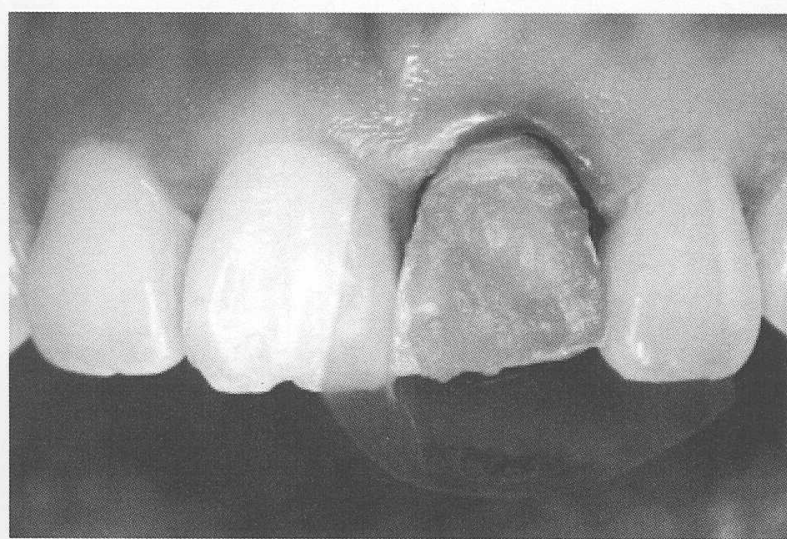

Figure 6: An even layer of etchant gel (36\% phosphoric acid) was placed over the preparation. The acid was left for 15 seconds before rinsing it off with water.

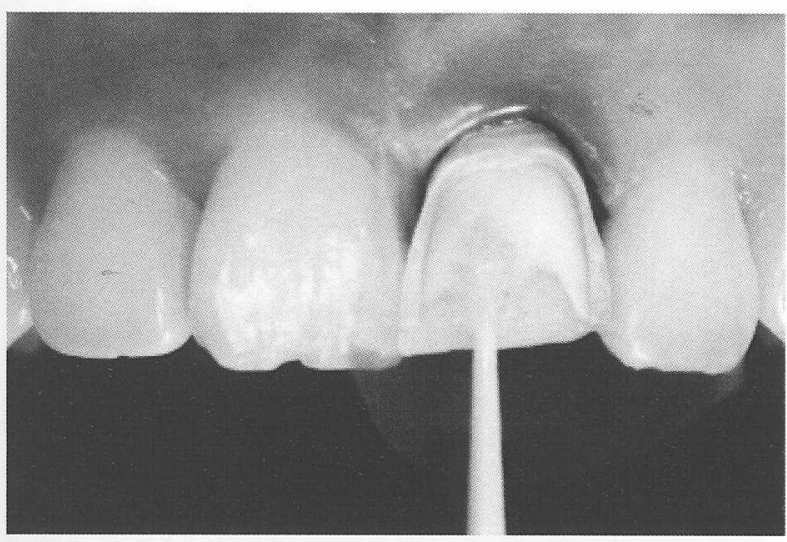

Figure 7: A layer of Prime \& Bond $\mathrm{NT}^{\circledR}$ was applied to the surface and was left for 20 seconds. A slow stream of air was used to blow the solvent off. It was then light cured for 10 seconds.

composite while it is still pliable and before light curing.

7. Ceram.X Duo enamel shade (E2) was placed at the labial surface (Fig. 10) and contoured with a flat plastic instrument. Then it was light cured

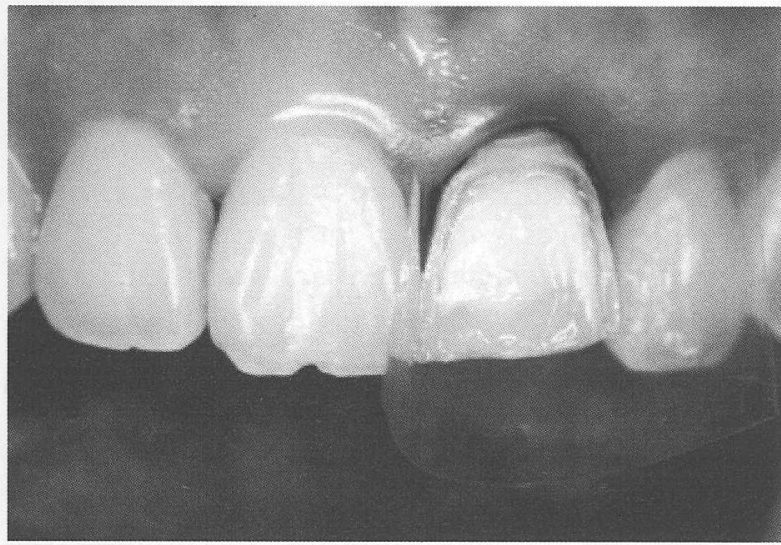

Figure 8: A thin layer of Ceram.X Duo dentine shade (D1) was placed on the prepared labial surface.

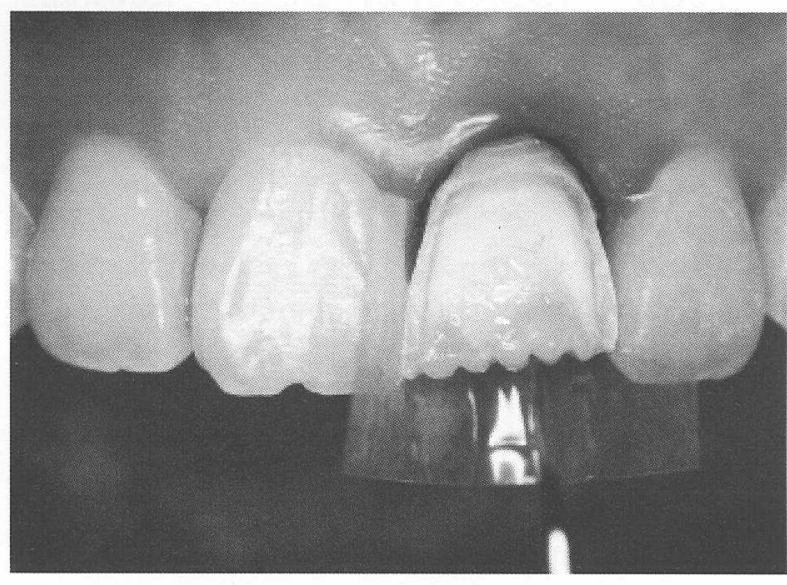

Figure 9: The Ceram.X Duo dentine shade was sculpted with flat plastic at the incisal level to form the mammelon-like effect.

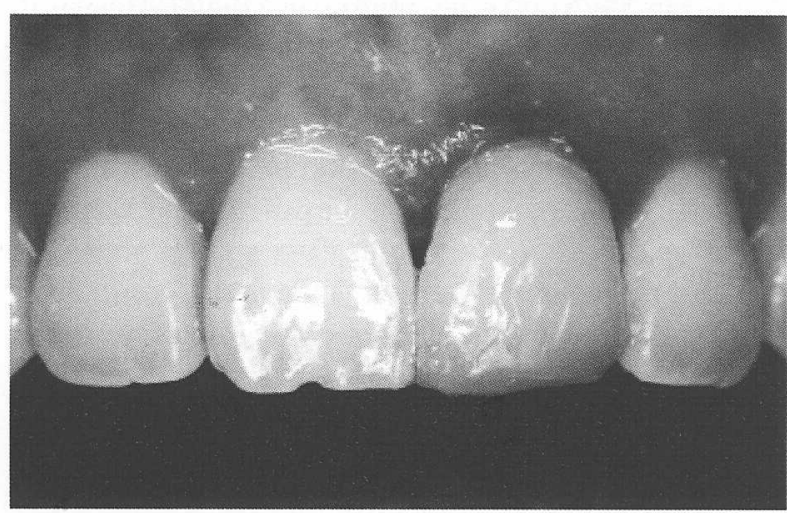

Figure 10: Ceram.X Duo enamel shade (E2) was placed at the labial surface.

for 10 seconds followed by removal of the retraction cord.

8. The surface anatomy was contoured using multifluted carbide finishing bur (Komet no. H22 ALGK.314.016, Gebr, Brasseler, Germany) 
and tapered fine diamond bur (Komet no. 845.314.012, Gebr, Brasseler, Germany) (Fig. 11 \& 12). Clinical tip: Use the multifluted carbide finishing bur to blend the restoration into the tooth and to characterize the restoration by creating lobes and labial lines angle. Use a flame shaped fine diamond bur to accentuate the surface anatomy and natural texture of the labial surface.

9. Restoration was finished using Enhance ${ }^{\circledR}$ (DENTSPLY DeTrey, Konstanz, Germany) finishing system (Fig. 13). Clinical tip: Use light pressure as it leaves a smooth surface without removing the bulk material. The greater the pressure, the more material is removed.

10. Subsequently, the restoration was polished with PoGoTM (DENTSPLY DeTrey, Konstanz, Germany) polishing system (Fig. 14). Clinical tip: Start with light pressure. Then decrease pressure to 'feather-like' touch to bring out the highest luster. Disc polisher should be in contact with the restoration.

11. Tooth 21 was veneered with Ceram.X Duo with the contour and surface anatomy simulating to that of contralateral tooth (Fig. 15 \& 16).

\section{DISCUSSION}

This case has illustrated the use of Ceram.X Duo, a nano ceramic composite restorative material in the construction of a direct veneer restoration. This material exhibits mechanical and physical properties that are more superior to those of their predecessors $(6,7)$. The nano ceramic composite material used in this case contains 3 types of fillers ie conventional glass filler $( \pm 1 \mu \mathrm{m})$, nano fillers $( \pm 10 \mathrm{~nm})$ and organically modified ceramic nano-particles (2-3 $\mathrm{nm})$. This nano ceramic technology has the advantages of ideal handling, excellent biocompatibility (8) and high fracture toughness as claimed by the manufacturer. In general, composites with smaller filler particles exhibit an improved polishing capacity and have greater surface smoothness and less general wear when compared to larger filler particles $(8,9)$.

Although resin composite veneers are known to be susceptible to discoloration, wear and marginal fractures in the long term, they have an advantage of being simple and comfortable procedure that takes just one visit, as well as cost-effective. Innovative techniques together with new nano ceramic restorative material enable the production of excellent aesthetics, acceptable durability with minimal tooth preparation.

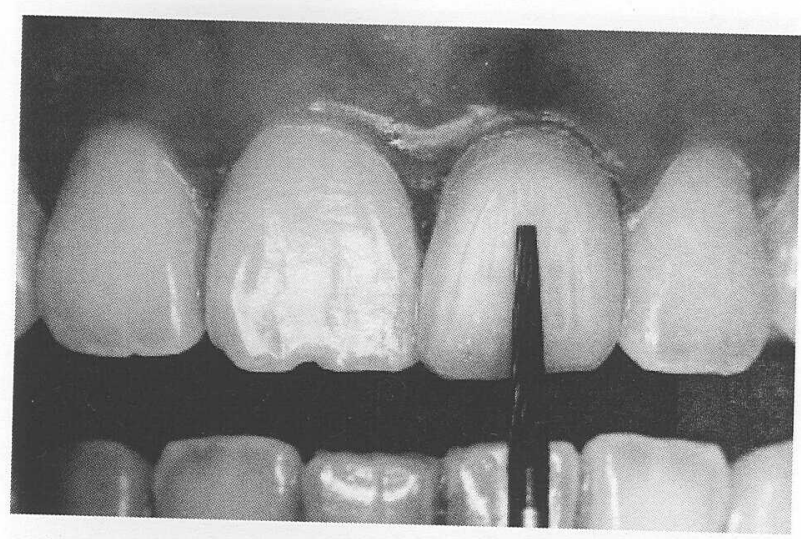

Figure 11: A multifluted carbide finishing bur was used to restore the surface anatomy.

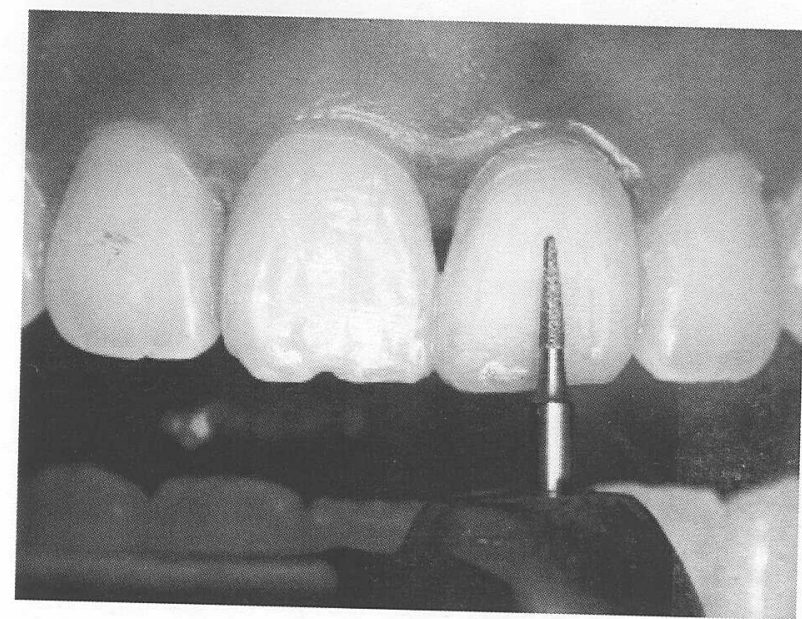

Figure 12: A tapered fine grit diamond bur was used to accentuate the surface anatomy and natural texture of the labial surface.

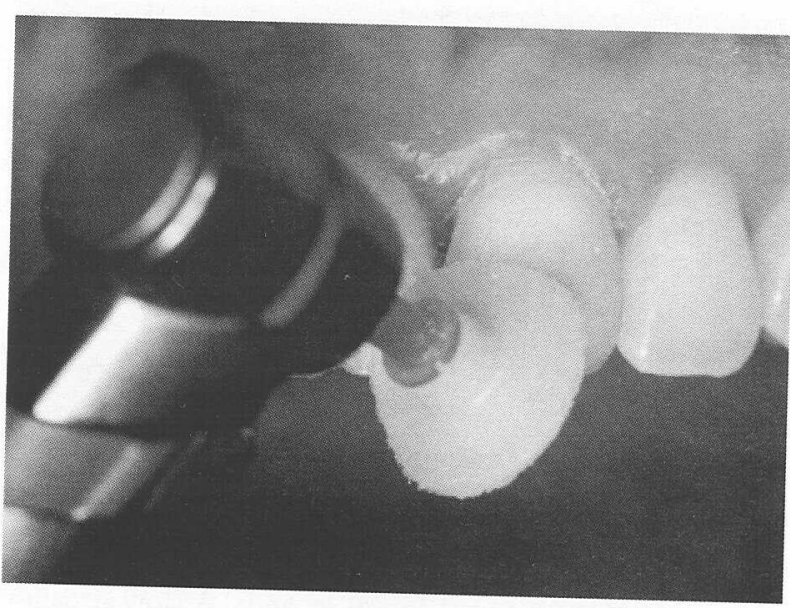

Figure 13: The Enhance ${ }^{\circledR}$ disc was used to finish the middle and incisal third of the restoration. Enhance ${ }^{\circledR}$ finishing cup was used to finish the restoration mainly at the cervical third. 


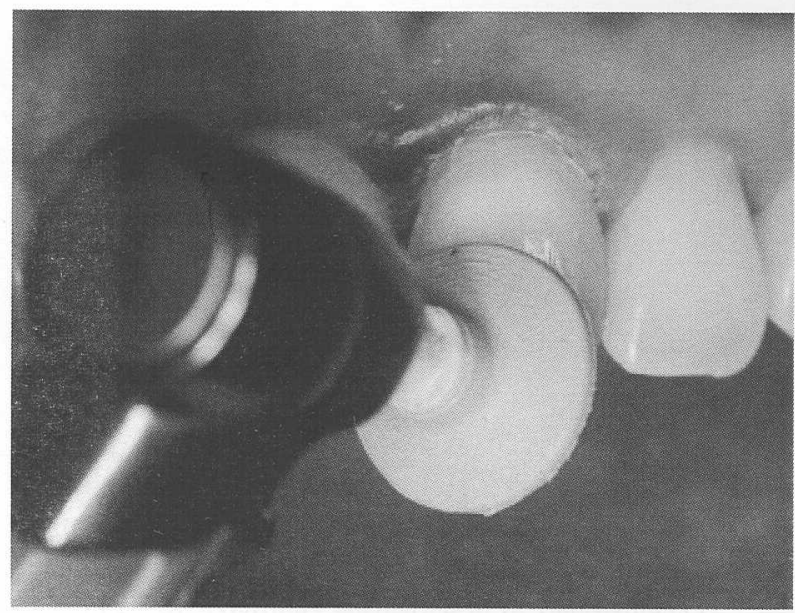

Figure 14: The smooth and defect-free restoration was polished with $\mathrm{PoGo}{ }^{\mathrm{TM}}$ disc. The cervical restoration was further polished with PoGo ${ }^{\mathrm{TM}}$ cup.

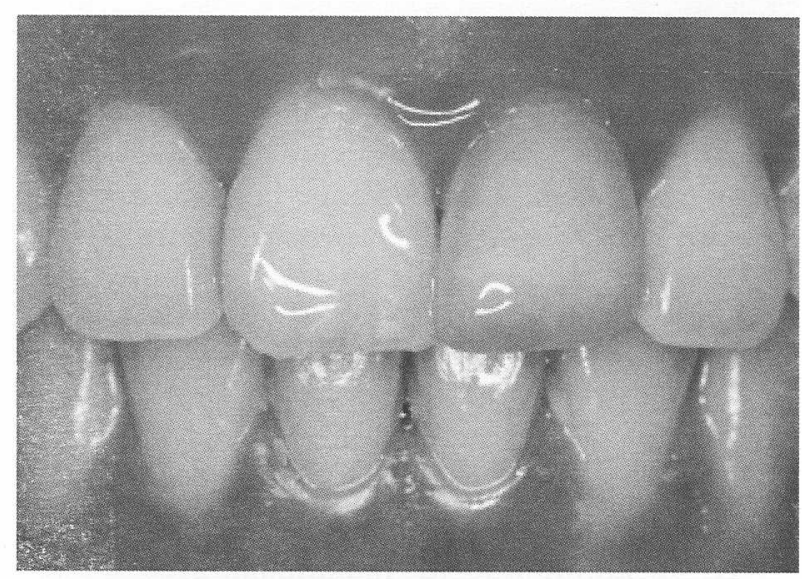

Figure 15: Postoperative appearance of Ceram.X Duo restoration.

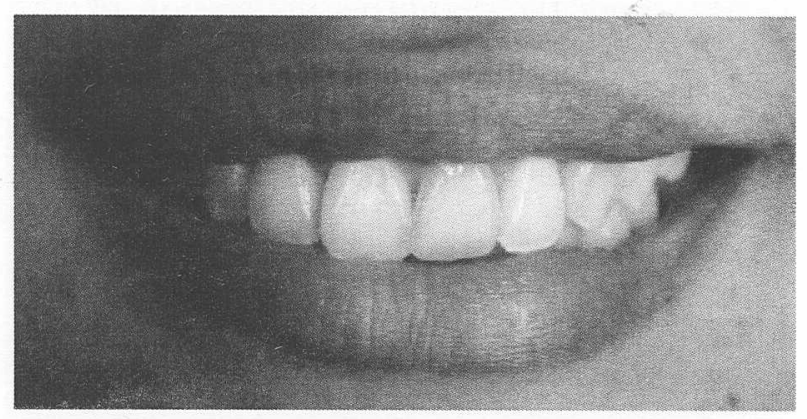

Figure 16: Ceram.X Duo restoration at 1-week recall.

\section{CONCLUSION}

This case report has shown that direct veneer composite resin restoration is a worthy conservative treatment option in restoring discolored and poorly contoured tooth to excellent aesthetic and contour. The evolution of nano ceramic restorative materials together with simple clinical techniques has made the effort to deliver anterior aesthetics and designing an attractive smile possible.

\section{REFERENCES}

1. Goldstein RE. Survey of patient attitudes toward current esthetic procedures. J Prosthet Dent 1984; 52: 775-780.

2. Rosenstiel SF, Land MF, Fujimoto J. Contemporary fixed prosthodontics. $4^{\text {th }}$ edn. Mosby Elsevier 2006.

3. Nakabayashi N, Kojima K, Masuhara E. The promotion of adhesion by infiltration of monomers into tooth substrates. J Biomed Mat Res 1982; 16: 265-73.

4. Van Meerbeek B, Vanherle G, Lambrechts P, et al. Dentin- and enamel-bonding agents. Curr Op Dent 1992; 2: 117-27.

5. Pashley DH, Ciucchi B, Sano $\mathrm{H}$, et al. Permeability of dentin to adhesive agents. Quint Int 1993; 24: 618-31.

6. Schirrmeister JF, Huber K, Hellwig E, Hahn P. Two-year evaluation of a new nano-ceramic restorative material. Clin Oral Investig 2006; 10(3): 181-6.

7. Makoto M. Clinical application of the newly designed resin composite including nanofillers "Ceram.X". Adhes Dent 2005; 23: 118-21.

8. Moszner N, Klapdohr S. Nanotechnology for dental composite. Int J Nanotech 2004; 1: 130 56.

9. van Noort R. Introduction to dental materials. $3^{\text {rd }}$ edn. Mosby 2007. 